\title{
Malignant Respiratory Tract Neoplasm
}

National Cancer Institute

\section{Source}

National Cancer Institute. Malignant Respiratory Tract Neoplasm. NCI Thesaurus. Code C4571.

A primary or metastatic malignant neoplasm that affects the lung parenchyma, bronchial tree, or trachea. Representative examples include lung carcinoma, carcinoid tumor, lung lymphoma, lung sarcoma, and tracheal carcinoma. 